\title{
Heat-Not-Burn Tobacco Products: The Devil in Disguise or a Considerable Risk Reduction?
}

\author{
Dirk W. Lachenmeier ${ }^{1,2}$, Peter Anderson ${ }^{5}$, Jürgen Rehm ${ }^{1,3,4}$ \\ ${ }^{1}$ Epidemiological Research Unit, Technische Universität Dresden, Klinische Psychologie and Psychotherapie, Dresden, Germany \\ ${ }^{2}$ Chemisches und Veterinäruntersuchungsamt (CVUA) Karlsruhe, Karlsruhe, Germany \\ ${ }^{3}$ Institute for Mental Health Policy Research, Centre for Addiction and Mental Health (CAMH), Toronto, Canada \\ ${ }^{4}$ Dalla Lana School of Public Health and Department of Psychiatry, University of Toronto \\ ${ }^{5}$ Institute of Health and Society, Newcastle University, Newcastle, England
}

\begin{abstract}
Background: Heat-not-burn (HNB) tobacco products are not burnt but instead are inserted into a tobacco-heating system, which heats the tobacco at temperatures below that required to initiate combustion. This mechanism potentially results in significantly reduced concentrations of heat-generated toxicants in the inhalable aerosol.

Method: The margin of exposure (MOE) approach was applied for quantitative risk assessment. The MOE is defined as the ratio between the toxicological threshold and the estimated human intake of the same compound. The higher the MOE, the lower the risk of a compound.

Findings: The MOEs were increased by factors of 3 to 415 for the most toxic compounds in tobacco smoke, comparing use of HNB with smoking conventional tobacco products. The combined MOE for all compounds was increased 23-fold, excluding nicotine, or 10-fold including nicotine. Thus, the overall risk for cumulative toxic effects was markedly lower for HNB products.

Conclusions: HNB tobacco reduced the risk of exposure to 9 out of the 20 most toxic compounds in tobacco beyond an MOE threshold of 10,000. While our results show that use of HNB products leads to a considerable risk reduction compared to conventional tobacco, the products cannot be considered completely "risk-free" due to risk of exposure to the remaining toxicants with MOE below the threshold.
\end{abstract}

Heat-not-burn (HNB) tobacco products were first introduced for sale in 1988. They are now marketed as the tobacco industry's response to non-tobacco-containing nicotine delivery systems, such as electronic cigarettes (Caputi, 2017). HNB cigarettes are not burnt but instead are inserted into a tobacco-heating system, which heats the tobacco at temperatures below those required to initiate combustion (Smith et al., 2017). This mechanism potentially results in significantly reduced concentrations of heat-generated toxicants in the inhalable aerosol (Smith et al., 2017). Some European countries have reported exponential growth of sales of HNB cigarettes since 2016 (Liu et al., 2018).

There is a lack of independent scientific studies of HNB tobacco products. Only limited in vivo and in vitro research about the effects of HNB cigarettes is available (Phillips et al., 2018; Titz et al., 2018; Titz et al., 2015; Brossard et al., 2017; Picavet, Haziza, Lama, Weitkunat, \& Lüdicke, 2015; Kamada, Yamashita, \& Tomioka, 2016). Regarding the smoke composition, only a few recent studies were without industry affiliation (Li et al., 2018; Auer, Concha-Lozano, Jacot-Sadowski, Cornuz, \& Berthet, 2017; Mallock et al., 2018; see also Jenssen, Walley, \& McGrath-Morrow [2017] for a critical review of industry-financed HNB studies). While similar industry studies (Schaller et al., 2016; Jaccard et al., 2017) typically concluded that a $90 \%$ reduction of harmful and potentially harmful constituents occurred with use of HNB products as compared to traditional tobacco products, Auer et al. (2017) found lower reductions for some compounds, ranging from only an $18 \%$ reduction for acrolein to an increase of $295 \%$ for acenaphthene. In other studies without industry affiliation, Mallock et al. (2018) confirmed reduced aldehyde levels (80-95\%) and reduced volatile organic compounds (97-99\%), and $\mathrm{Li}$ et al. (2018) determined $80 \%$ lower releases for some compounds. However, none of these studies provided a quantitative risk assessment; that is, they typically neglected the different potencies of the compounds, which may differ by several orders of magnitude, and their impact on health risks. Currently available integrated modelling has concluded that

Correspondence: Dirk W. Lachenmeier, PhD, Chemisches und Veterinäruntersuchungsamt (CVUA) Karlsruhe, Weissenburger Strasse 3, 76187 Karlsruhe, Germany. Phone +49721926 5434. Fax +49721926 3549 (Lachenmeier@web.de) Financial support: No funding to report.

Declaration of interest: The authors have no conflict of interest to declare.

Keywords: tobacco products, smoking, cigarettes, nicotine, risk assessment, carcinogens 
HNB devices have lower cancer potencies than tobacco smoke by at least one order of magnitude (Stephens, 2017). However, in this model, only a sub-group of compounds, not including acenaphthene, was considered.

Our study provides a comprehensive quantitative comparative risk assessment of tobacco smoke constituents, using the margin of exposure (MOE) approach, comparing HNB to conventional tobacco products. The MOE is a standard methodology for toxicological risk assessment (for a description, see European Food Safety Authority [2005] and Crump, Allen, \& Faustman [1995], and for application in tobacco field, see Baumung, Rehm, Franke, \& Lachenmeier [2016] and Hahn et al. [2014]).

\section{Method}

The methodology for quantitative risk assessment is based on a previous study for risk assessment of tobacco smoke constituents (Baumung et al., 2016; Lachenmeier \& Rehm, 2015). The selection of compounds is restricted to those with an MOE below 10,000 according to Baumung et al. (2016). The usual threshold for genotoxic carcinogens is 10,000 (e.g., European Food Safety Authority, 2005; European Commission, 2008). In addition, acenaphthene was included due to the finding by Auer et al. (2017) that it is contained in higher amounts in HNB than in conventional tobacco products. An MOE is defined as the ratio between the toxicological threshold and the estimated human intake of the same compound. Toxicological thresholds - typically benchmark dose lower confidence limits - and a description of the endpoints used for toxicological thresholds were taken from Baumung et al. (2016). The exposures for smoking 20 cigarettes per day were estimated using probabilistic Monte Carlo simulation based on average and standard deviations reported in three studies comparing HNB with conventional cigarettes (Auer et al., 2017; Schaller et al., 2016; Jaccard et al., 2017). Higher MOEs indicate lower risk. The MOE was calculated using the software package @RISK for Excel Version 5.5.0.

The combined margin of exposure (MOET) is calculated as

$$
\frac{1}{\frac{1}{M O E_{1}}+\frac{1}{M O E_{2}}+\cdots}
$$

MOET can be interpreted as a rough estimate of additive cumulative exposure, disregarding any differences in target site, toxicological mechanism, or potential synergistic interactions of compounds. Descriptive statistics of MOET results are provided as box plots, with the box being determined by the 25th and 75th percentiles. The whiskers are determined by the 5th and 95th percentiles. First and 99th percentiles are marked by $\mathrm{x}$, while the minimum and maximum are marked with dashes.

\section{Results}

Except for acenaphthene, the MOEs of all compounds were increased (i.e., indicating less risk) by factors of 3-9 for acetaldehyde, ammonia, arsenic, chromium, catechol, formaldehyde, and pyridine, comparing HNB with conventional tobacco. For the rest of the compounds (see Table 1), the increase was above a factor of 10 , up to 415 fold for isoprene. For HNB, the MOE of several compounds (acenaphthene, benzene, chromium, m/p-cresol, ethylene oxide, isoprene, 4-(methylnitrosamino)-1-(3-pyridyl)-1butanone (NNK), N-nitrosonornicotine (NNN), quinoline, and styrene) exceeded the threshold of 10,000, while for conventional tobacco this was the case only for acenaphthene.

The combined $\mathrm{MOE}_{\mathrm{T}}$ for all compounds was increased 23fold for HNB compared to conventional tobacco smoking without nicotine (see Figure 1), or 10-fold with nicotine. Thus, the overall risk for cumulative toxic effects was markedly lower with use of HNB products compared to conventional tobacco products.

\section{Discussion}

HNB tobacco combined with an electronic tobacco-heating system reduced the risk of exposure to 9 of the 20 most toxic compounds in tobacco beyond a MOE threshold of 10,000, which is generally used as relevant to public health (European Commission, 2008). Acenaphthene, the only compound found in higher concentrations in $\mathrm{HNB}$, has a very high MOE in both $\mathrm{HNB}$ and conventional tobacco and can be considered unlikely to pose any significant health risk to the consumer. Our findings corroborate the study of Mallock et al. (2018) who showed comparable nicotine yield between HNB and typical combustible cigarettes, but substantially reduced levels of aldehydes and volatile organic compounds. The differences of the Auer et al. (2017) study may derive from an unvalidated analytical methodology (e.g., Maeder \& Peitsch, 2017).

While our results show that HNB leads to a considerable risk reduction compared to conventional tobacco, the risk reduction is not complete and several compounds remain with MOE values considered to be at risk-entailing levels. Limitations in the risk assessment also include substances not currently being analyzed, such as pyrolysis products of plastics and other ingredients and additives unique to HNB (Mallock et al., 2018; Davis, Williams, \& Talbot, 2018). The risk assessment also only considers isolated chemical compounds and does not include other acute and chronic effects-for example, respiratory or cardiovascular effects caused by other potential mechanisms such as temperature or other physical effects of the aerosol.

Nevertheless, HNB tobacco might be a reasonable choice for risk reduction by heavy smokers otherwise unwilling or unable to stop smoking. From a public health point of view, what is key is whether HNB tobacco will be used by existing heavy smokers unwilling or unable to stop, whether it will prevent existing smokers from stopping completely, and whether it will attract new non-smoking customers. 
Table 1

Average margin of exposure (MOE) for tobacco constituents comparing exposure from heat-not-burn emissions using tobaccoheating systems with conventional tobacco smoke

\begin{tabular}{lll}
\hline Constituent & $\begin{array}{c}\text { MOE for heat-not-burn } \\
\text { emissions using tobacco } \\
\text { heating systems }\end{array}$ & $\begin{array}{c}\text { MOE for conventional } \\
\text { cigarette smoke }\end{array}$ \\
\hline HCN & $557^{*}$ & 6 \\
1,3-Butadiene & 3425 & 12 \\
Acrolein & 79 & 6 \\
Acrylonitrile & 2269 & 15 \\
Isoprene & 149743 & 361 \\
Formaldehyde & 358 & 40 \\
Acetaldehyde & 1197 & 326 \\
Cadmium compounds & $3064 *$ & 9 \\
Catechol & 994 & 165 \\
Benzene & 14292 & 93 \\
Chromium & $37427 *$ & $9846^{*}$ \\
Ammonia & 1481 & 533 \\
Arsenic & $9532 *$ & 1441 \\
Quinoline & $42123 *$ & 1070 \\
Pyridine & 2071 & 432 \\
Styrene & 55085 & 2197 \\
4-(Methylnitrosamino)-1-(3-pyridyl)-1-butanone (NNK) & 10140 & 278 \\
m/p-Cresol & $98435 *$ & 573 \\
Ethylene oxide & 64749 & 499 \\
N-Nitrosonornicotine (NNN) & 66463 & 2910 \\
Acenaphthene & 5634244 & 12600430 \\
Nicotine & 36 & 21 \\
MOE excluding nicotine & 21 & 0.9 \\
MOET including nicotine & 8 & 0.8 \\
\hline * Marked values must be treated as worst-case scenarios (non-quantifiable concentrations were set at the limit of quantification)
\end{tabular}

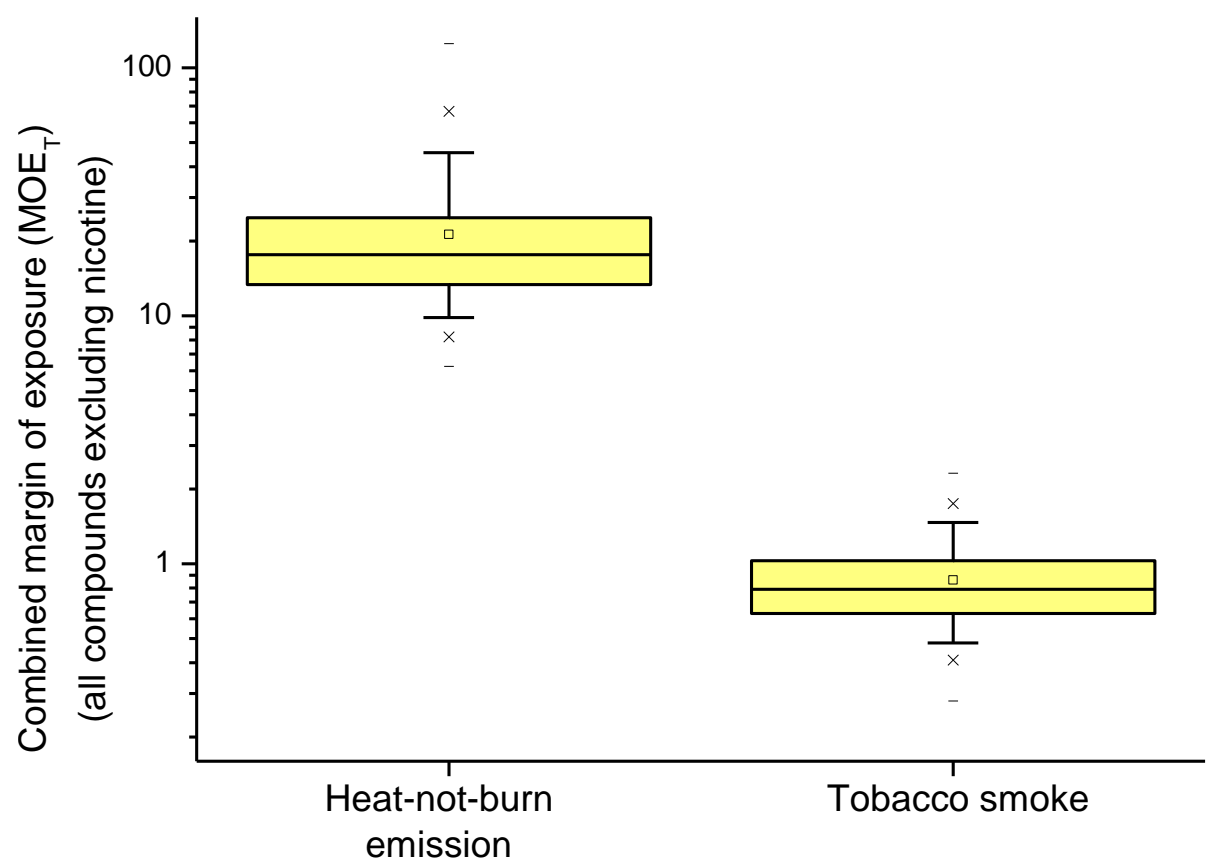

Figure 1. Combined margin of exposure for tobacco constituents excluding nicotine for daily smokers (20 cigarettes). 


\section{References}

Auer, R., Concha-Lozano, N., Jacot-Sadowski, I., Cornuz, J., \& Berthet, A. (2017). Heat-not-burn tobacco cigarettes: Smoke by any other name. JAMA Internal Medicine, 177(7), 1050-1052.

Baumung, C., Rehm, J., Franke, H., \& Lachenmeier, D. W. (2016). Comparative risk assessment of tobacco smoke constituents using the margin of exposure approach: The neglected contribution of nicotine. Scientific Reports, 6, 35577.

Brossard, P., Weitkunat, R., Poux, V., Lama, N., Haziza, C., Picavet, P., . . . Lüdicke, F. (2017). Nicotine pharmacokinetic profiles of the Tobacco Heating System 2.2, cigarettes and nicotine gum in Japanese smokers. Regulatory Toxicology and Pharmacology, 89, 193-199.

Caputi, T. L. (2017). Industry watch: Heat-not-burn tobacco products are about to reach their boiling point. Tobacco Control, 26(5), 609-610.

Crump, K. S., Allen, B., \& Faustman, E. (1995). The use of the benchmark dose approach in health risk assessment. Washington, D.C.: Risk Assessment Forum, US Environmental Protection Agency.

Davis, B., Williams, M., \& Talbot, P. (2018). iQOS: Evidence of pyrolysis and release of a toxicant from plastic. Tobacco Control [Epub ahead of print]. doi:10.1136/tobaccocontrol-2017-054104

European Commission. (2008). Risk assessment methodologies and approaches for mutagenic and carcinogenic substances. Brussels, Belgium: European Commission.

European Food Safety Authority. (2005). Opinion of the Scientific Committee on a request from EFSA related to a harmonised approach for risk assessment of substances which are both genotoxic and carcinogenic. EFSA Journal, 3(10), 282.

Hahn, J., Monakhova, Y. B., Hengen, J., KohlHimmelseher, M., Schüssler, J., Hahn, H., . . . Lachenmeier, D. W. (2014). Electronic cigarettes: Overview of chemical composition and exposure estimation. Tobacco Induced Diseases, 12(1), 23.

Jaccard, G., Djoko, D. T., Moennikes, O., Jeannet, C., Kondylis, A., \& Belushkin, M. (2017). Comparative assessment of HPHC yields in the Tobacco Heating System THS2. 2 and commercial cigarettes. Regulatory Toxicology and Pharmacology, 90, 1-8.

Jenssen, B. P., Walley, S. C., \& McGrath-Morrow, S. A. (2017). Heat-not-burn tobacco products: Tobacco industry claims no substitute for science. Pediatrics, e20172383.

Kamada, T., Yamashita, Y., \& Tomioka, H. (2016). Acute eosinophilic pneumonia following heat-not-burn cigarette smoking. Respirology Case Reports, 4(6), $\mathrm{e} 00190$.

Lachenmeier, D. W., \& Rehm, J. (2015). Comparative risk assessment of alcohol, tobacco, cannabis and other illicit drugs using the margin of exposure approach. Scientific Reports, 5, 8126.

Li, X., Luo, Y., Jiang, X., Zhang, H., Zhu, F., Hu, S., . . Pang, Y. (2018). Chemical analysis and simulated pyrolysis of Tobacco Heating System 2.2 compared to conventional cigarettes. Nicotine \& Tobacco Research [Epub ahead of print]. doi:10.1093/ntr/nty005

Liu, X., Lugo, A., Spizzichino, L., Tabuchi, T., Gorini, G., \& Gallus, S. (2018). Heat-not-burn tobacco products are getting hot in Italy. Journal of Epidemiology, 28(5), 274-275.

Maeder, S., \& Peitsch, M. C. (2017). Perplexing conclusions concerning heat-not-burn tobacco cigarettes. JAMA Internal Medicine, 177(11), 16981699.

Mallock, N., Böss, L., Burk, R., Danziger, M., Welsch, T., Hahn, H., . . Hutzler, C. (2018). Levels of selected analytes in the emissions of "heat not burn" tobacco products that are relevant to assess human health risks. Archives of Toxicology, 92(6), 2145-2149.

Phillips, B. W., Schlage, W. K., Titz, B., Kogel, U., Sciuscio, D., Martin, F., . . Veljkovic, E. (2018). A 90-day OECD TG 413 rat inhalation study with systems toxicology endpoints demonstrates reduced exposure effects of the aerosol from the carbon heated tobacco product version 1.2 (CHTP1. 2) compared with cigarette smoke. I. Inhalation exposure, clinical pathology and histopathology. Food and Chemical Toxicology, 116, 388-413.

Picavet, P., Haziza, C., Lama, N., Weitkunat, R., \& Lüdicke, F. (2015). Comparison of the pharmacokinetics of nicotine following single and ad libitum use of a tobacco heating system or combustible cigarettes. Nicotine \& Tobacco Research, 18(5), 557-563.

Schaller, J. P., Keller, D., Poget, L., Pratte, P., Kaelin, E., McHugh, D., ... Yerly, M. (2016). Evaluation of the Tobacco Heating System 2.2. Part 2: Chemical composition, genotoxicity, cytotoxicity, and physical properties of the aerosol. Regulatory Toxicology and Pharmacology, 81, S27-S47.

Smith, M. R., Clark, B., Lüdicke, F., Schaller, J. P., Vanscheeuwijck, P., Hoeng, J., \& Peitsch, M. C. (2016). Evaluation of the Tobacco Heating System 2.2. Part 1: Description of the system and the scientific assessment program. Regulatory Toxicology and Pharmacology, 81, S17-S26.

Stephens, W. E. (2018). Comparing the cancer potencies of emissions from vapourised nicotine products including e-cigarettes with those of tobacco smoke. Tobacco Control, 27, 10-17.

Titz, B., Boué, S., Phillips, B., Talikka, M., Vihervaara, T., Schneider, T., . . Schlage, W. K. (2015). Effects of cigarette smoke, cessation, and switching to two heatnot-burn tobacco products on lung lipid metabolism in C57BL/6 and Apoe-/- Mice-An integrative systems toxicology analysis. Toxicological Sciences, 149(2), 441-457.

Titz, B., Kogel, U., Martin, F., Schlage, W. K., Xiang, Y., Nury, C., . . . Dulize, R. (2018). A 90-day OECD TG 413 rat inhalation study with systems toxicology endpoints demonstrates reduced exposure effects of the aerosol from the carbon heated tobacco product version 1.2 (CHTP1. 2) compared with cigarette smoke. II. Systems toxicology assessment. Food and Chemical Toxicology, 115, 284-301. 